\title{
Observations of MIRA stars with the IOTA/FLUOR interferometer and comparison with MIRA star models
}

Karl-Heinz Hofmann, Udo Beckmann, Thomas Bloecker, Vincent Coude du Foresto, Marc G. Lacasse, et al.

Karl-Heinz Hofmann, Udo Beckmann, Thomas Bloecker, Vincent Coude du Foresto, Marc G. Lacasse, Rafael Millan-Gabet, Sebastien Morel, B. Pras, Cyril Ruilier, Dieter Schertl, Michael Scholz, Victor Shenavrin, Wesley A. Traub, Gerd Weigelt, Markus Wittkowski, Boris Yudin, "Observations of MIRA stars with the IOTA/FLUOR interferometer and comparison with MIRA star models," Proc. SPIE 4006, Interferometry in Optical Astronomy, (5 July 2000); doi: 10.1117/12.390270 


\title{
Observations of Mira stars with the IOTA/FLUOR interferometer and comparison with Mira star models
}

\author{
K.-H. Hofmann ${ }^{a}$, U. Beckmann ${ }^{a}$, T. Blöcker ${ }^{a}$, V. Coude du Foresto ${ }^{b}$, M. Lacasse $^{c}$, \\ R. Millan-Gabet ${ }^{c}$, S. Morel ${ }^{c}$, B. Pras ${ }^{c}$, C. Ruilier ${ }^{b}$, D. Schertl ${ }^{a}$, M. Scholz ${ }^{d}$, V. Shenavrin ${ }^{e}$, \\ W. Traub ${ }^{c}$, G. Weigelt ${ }^{a}$, M. Wittkowski ${ }^{a}$, B. Yudin ${ }^{e}$ \\ ${ }^{a}$ MPI für Radioastronomie, Auf dem Huegel 69, 53121 Bonn, Germany \\ ${ }^{b}$ Observatoire de Paris-Meudon, 5 place Jules Janssen, \\ 92195 Meudon Cedex, France \\ ${ }^{c}$ Harvard-Smithsonian Center for Astrophysics, 60 Garden Street, \\ Cambridge, Massachusetts 02138, USA \\ ${ }^{d}$ Institut für Theoretische Astrophysik der Universitaet Heidelberg,Tiergartenstrasse 15, \\ 69121 Heidelberg, Germany \\ ${ }^{e}$ Sternberg Astronomical Institute, Universitetskii pr. 13, \\ 119899 Moscow, Russia.
}

\begin{abstract}
We present K-band observations of five Mira stars with the IOTA interferometer. The interferograms were obtained with the FLUOR fiber optics beam combiner which provides high-accuracy visibility measurements in spite of timevariable atmospheric conditions. For the Mira stars X Oph, R Aql, RU Her, R Ser, and V CrB we derived the uniform-disk diameters 11.7 mas, 10.9 mas, 8.4 mas, 8.1 mas, and 7.9 mas ( \pm 0.3 mas), respectively. Simultaneous photometric observations yielded the bolometric fluxes. The derived angular Rosseland radii and the bolometric fluxes allowed the determination of effective temperatures. For instance, the effective temperature of R Aql was determined to be $3072 \mathrm{~K} \pm 161 \mathrm{~K}$. A Rosseland radius for $\mathrm{R}$ Aql of $250 \mathrm{R}_{\odot} \pm 63 \mathrm{R}_{\odot}$ was derived from the angular Rosseland radius of $5.5 \mathrm{mas} \pm 0.2 \mathrm{mas}$ and the HIPPARCOS parallax of 4.73 mas \pm 1.19 mas. The observations were compared with theoretical Mira star models ${ }^{1,2}\left(\mathrm{D} / \mathrm{P}\right.$ model Rosseland radius $=255 \mathrm{R}_{\odot}$; measured $\mathrm{R}$ Aql Rosseland radius $\left.=250 R_{\odot} \pm 63 R_{\odot}\right)$.
\end{abstract}

Keywords: interferometry, Mira variables

\section{INTRODUCTION}

The resolution of large optical telescopes and interferometers is high enough to resolve the stellar disk of nearby $\mathrm{M}$ giant stars, to reveal photospheric asymmetries and surface structures, and to study the dependence of the diameter on the wavelength, variability phase, and cycle. Previous speckle or long-baseline interferometry observations were, for example, reported in Refs. 3-10. Theoretical studies (e.g. Refs. 1-2 and 11-13) show that accurate monochromatic diameter measurements can significantly improve our understanding of $\mathrm{M}$ giant atmospheres. With the IOTA interferometer a resolution of $\sim 9$ mas can be achieved with its largest baseline of $38 \mathrm{~m}$ in the K-band. The IOTA interferometer is located at the Smithsonian Institution's Whipple Observatory on Mount Hopkins in Arizona. A detailed description of IOTA can be found in Refs. 14 and 15. IOTA can be operated in the K-band with the FLUOR $^{16}$ fiber optics beam combiner. This beam combiner provides high-accuracy visibility measurements in spite of time-variable atmospheric conditions. The single-mode fibers in the beam combiner spatially filter the wavefronts corrugated by atmospheric turbulence (see Refs. 16 and 17).

\section{OBSERVATIONS}

The five Miras X Oph, R Aql, RU Her, R Ser, V CrB were observed with the IOTA interferometer on May 16, 17 and 18, 1999. The observations were carried out with the fiber optics beam combiner FLUOR in the K-band and with $38 \mathrm{~m}$ baseline. The interferograms are scanned by the delay line during the coherence time of the atmosphere. The OPD length of the scan is $\sim 100 \mu \mathrm{m}$. Approximately 100 scans per baseline were recorded. Several reference stars (Table 1) were observed for the calibration of the observations (see Ref. 17 for more details). The diameters of 
the reference stars were derived from the scale of stellar diameters at $\mathrm{K}$-magnitude $=0$ for giants by Dyck et al. ${ }^{18}$. The fringe visibility of the reference stars was $64 \%-94 \%$. Fig. 1 shows the obtained visibility functions of the five Mira stars together with uniform-disk fits. The errors of the derived Mira star diameters are 1-3\%.

Table 1. Observed data.

\begin{tabular}{|c|c|c|c|c|c|c|c|c|}
\hline Star & spectral type & $\begin{array}{c}P \\
{[\text { days }]}\end{array}$ & Date & $\overline{\Phi_{\text {vis }}}$ & $\begin{array}{l}B_{\mathrm{p}} \\
{[\mathrm{m}]}\end{array}$ & $V$ & $\begin{array}{l}\Theta_{\mathrm{UD}} \\
{[\mathrm{mas}]}\end{array}$ & reference stars \\
\hline $\mathrm{X} \mathrm{Oph}$ & M5e-M9e & 328 & $\begin{array}{l}99 \text { May } 17 \\
99 \text { May } 18 \\
99 \text { May } 18\end{array}$ & 0.71 & $\begin{array}{l}35.47 \\
34.75 \\
34.57\end{array}$ & $\begin{array}{l}0.2317 \pm 0.024 \\
0.2554 \pm 0.027 \\
0.2279 \pm 0.025\end{array}$ & $11.74 \pm 0.30$ & $\begin{array}{l}\text { HIP } 86742 \\
\text { HIP } 98337 \\
\text { HIP } 98438 \\
\text { HIP } 97278 \\
\text { HIP } 97278\end{array}$ \\
\hline $\mathrm{R} \mathrm{Aql}$ & M5e-M9e & 284 & $\begin{array}{l}99 \text { May } 17 \\
99 \text { May } 18\end{array}$ & 0.17 & $\begin{array}{l}35.42 \\
34.48\end{array}$ & $\begin{array}{l}0.2927 \pm 0.027 \\
0.3295 \pm 0.031\end{array}$ & $10.90 \pm 0.33$ & $\begin{array}{l}\text { HIP } 86742 \\
\text { HIP } 98337 \\
\text { HIP } 98438 \\
\text { HIP } 97278\end{array}$ \\
\hline RU Her & M6e-M9 & 484 & 99 May 17 & 0.07 & $\begin{array}{l}37.95 \\
37.73\end{array}$ & $\begin{array}{l}0.4768 \pm 0.017 \\
0.4769 \pm 0.017\end{array}$ & $8.36 \pm 0.20$ & $\begin{array}{l}\text { HIP } 71053 \\
\text { HIP } 78159\end{array}$ \\
\hline R Ser & M5e-M9e & 356 & 99 May 18 & 0.28 & 35.74 & $0.5467 \pm 0.016$ & $8.10 \pm 0.20$ & $\begin{array}{l}\text { HIP } 61658 \\
\text { HIP } 75530 \\
\text { HIP } 85934\end{array}$ \\
\hline V CrB & $\mathrm{C} 6,2 \mathrm{e}(\mathrm{N} 2 \mathrm{e})$ & 357 & 99 May 16 & 0.07 & $\begin{array}{l}37.78 \\
38.02\end{array}$ & $\begin{array}{l}0.5288 \pm 0.017 \\
0.5180 \pm 0.023\end{array}$ & $7.86 \pm 0.24$ & $\begin{array}{l}\text { HIP } 73555 \\
\text { HIP } 81833\end{array}$ \\
\hline
\end{tabular}

In Table 1 the calibrated visibilities and the derived uniform-disk diameters of the five Miras are listed, together with observational parameters (spectral type, variability period $P$, date of observation, variability phase $\Phi_{v i s}$, projected baseline length $B_{\mathrm{p}}$, calibrated visibilities $V$, derived uniform-disk diameters $\Theta_{\mathrm{UD}}$, and reference stars).

\section{COMPARISON OF THE OBSERVATIONS WITH MIRA STAR MODELS}

In this section we derive angular diameters from the measured visibilities by fitting different theoretical center-tolimb intensity variations (hereafter CLV) of different Mira star models (Bessel, Scholz and Wood $1996=$ BSW96 $^{1}$, Hofmann, Scholz and Wood $1998=$ HSW98 ${ }^{2}$ ). From these angular diameters and the bolometric flux, we derive effective temperatures. For R Aql a HIPPARCOS parallax is available which allows us to determine linear radii. The comparison of these measured stellar parameters with theoretical ones indicate whether any of the models are a fair representation of the observed Mira stars. All Mira star models used in this paper are from BSW96 (D and E series) and from HSW98 (P, M and O series). They were developed as possible representations of the prototype Mira variable o Ceti, and hence have periods $P$ very close to the 332 day period of this star; they differ in pulsation mode, assumed mass $M$ and assumed luminosity $L$; and the BSW96 models differ from the (more advanced) HSW98 models with respect to the pulsation modelling technique. The five models represent stars pulsating in the fundamental mode ( $f ; \mathrm{D}, \mathrm{P}$ and $\mathrm{M}$ models) or in the first-overtone mode ( $o ; \mathrm{E}$ and $\mathrm{O}$ models). Table 2 lists the properties of these Mira model series $\left(R_{\mathrm{p}}=\right.$ Rosseland radius of the non-pulsating parent star of the Mira variable $=$ distance from the "parent star's" center, at which the Rosseland optical depth $\tau_{\text {Ross }}$ equals unity, see BSW96 and HSW98; $T_{\text {eff }} \propto\left(L / R_{\mathrm{p}}^{2}\right)^{1 / 4}=$ effective temperature). Table 3 provides the link between the 22 abscissa values (model-phase combinations $\mathrm{m}$ ) in Figs. 2 and 3, and the models, and it additionally lists the variability phase, relative Rosseland and stellar K-band filter radius, and the effective temperature. We compare predictions of these models at different phases and cycles with our observations.

Monochromatic radius $R_{\lambda}$ and Rosseland radius $R$. We use the conventional stellar radius definition where the monochromatic radius $R_{\lambda}$ of a star at wavelength $\lambda$ is given by the distance from the star's center at which the optical depth equals unity $\left(\tau_{\lambda}=1\right)$. In analogy, the photospheric stellar radius $R$ (Rosseland radius) is given by the 

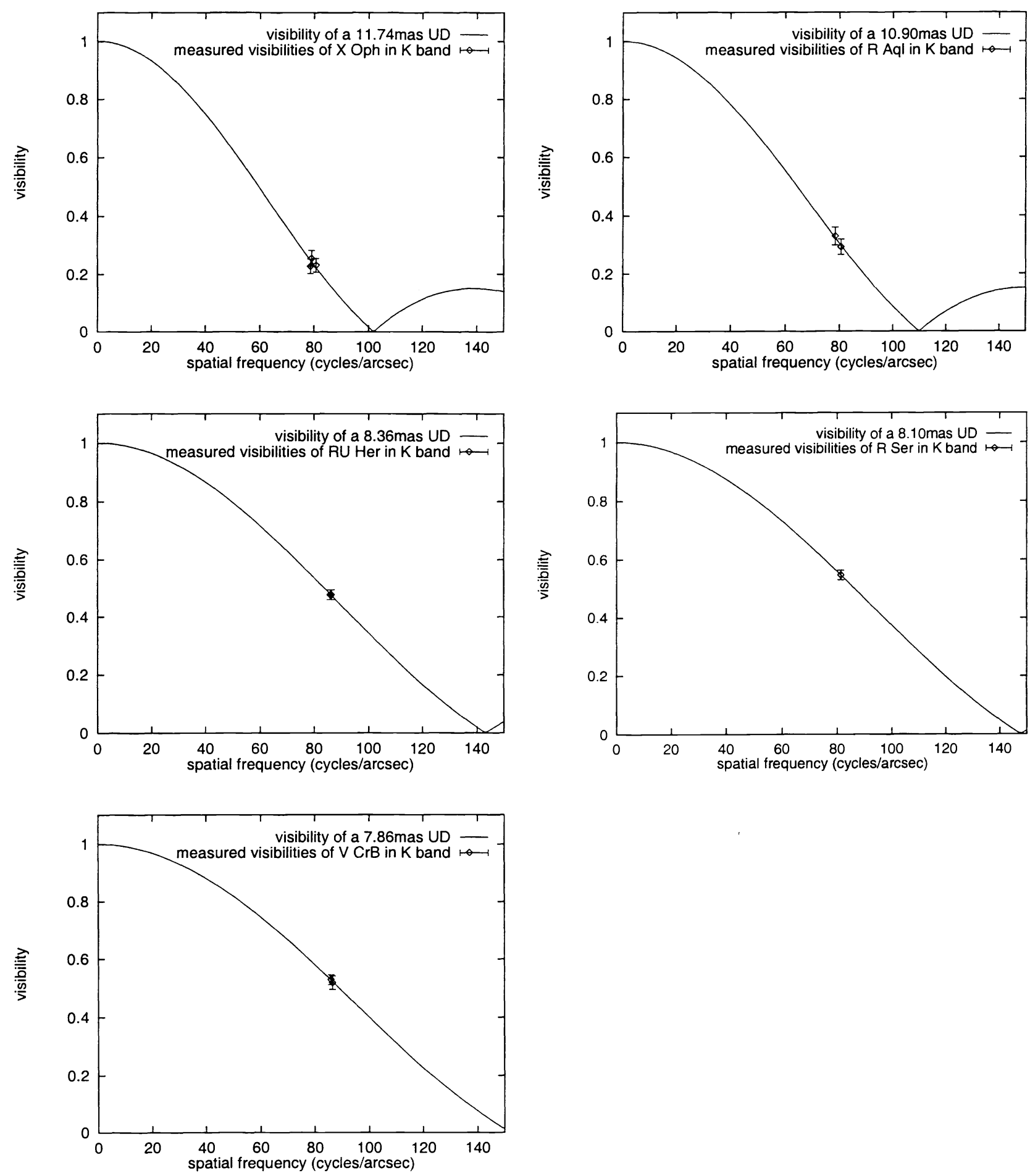

Figure 1. Uniform-disk fits (X Oph, R Aql, RU Her, R Ser, and V CrB). 
Table 2. Properties of Mira model series ${ }^{1,2}$ (see text)

\begin{tabular}{|c|c|c|c|c|c|c|}
\hline Series & Mode & $P($ days $)$ & $M / M_{\odot}$ & $L / L_{\odot}$ & $R_{\mathrm{p}} / R_{\odot}$ & $T_{\text {eff }} / \mathrm{K}$ \\
\hline $\mathrm{D}$ & $\mathrm{f}$ & 330 & 1.0 & 3470 & 236 & 2900 \\
$\mathrm{E}$ & $\mathrm{o}$ & 328 & 1.0 & 6310 & 366 & 2700 \\
$\mathrm{P}$ & $\mathrm{f}$ & 332 & 1.0 & 3470 & 241 & 2860 \\
$\mathrm{M}$ & $\mathrm{f}$ & 332 & 1.2 & 3470 & 260 & 2750 \\
$\mathrm{O}$ & $\mathrm{o}$ & 320 & 2.0 & 5830 & 503 & 2250 \\
\hline
\end{tabular}

Table 3. Link between the 22 abscissa values (model-phase combinations $\mathrm{m}$ ) in Figs. 2 and 3 , and the models. The variability phase $\Phi_{\text {vis }}$, the Rosseland radius $R$ and the K-band radius $R_{\mathrm{K}}$ in units of the parent star radius $R_{p}$, and the effective temperature $T_{\text {eff }}(R)$ associated to the Rosseland radius are additionally given.

\begin{tabular}{|l|l|l|l|l|l|}
\hline Model & $\Phi_{\text {vis }}$ & $R / R_{\mathrm{p}}$ & $R_{\mathrm{K}} / R_{\mathrm{p}}$ & $T_{\text {eff }}(R)$ & $\mathrm{m}$ \\
\hline D27520 & $1+0.0$ & 1.04 & 1.02 & 3020 & 1 \\
D27760 & $1+0.5$ & 0.91 & 0.90 & 2710 & 2 \\
D28760 & $2+0.0$ & 1.04 & 1.02 & 3030 & 3 \\
D28960 & $2+0.5$ & 0.91 & 0.91 & 2690 & 4 \\
E8300 & $0+0.83$ & 1.16 & 1.14 & 2330 & 5 \\
E8380 & $1+0.0$ & 1.09 & 1.10 & 2620 & 6 \\
E8560 & $1+0.21$ & 1.17 & 1.14 & 2610 & 7 \\
P71800 & $0+0.5$ & 1.20 & 1.04 & 2160 & 8 \\
P73200 & $1+0.0$ & 1.03 & 0.99 & 3130 & 9 \\
P73600 & $1+0.5$ & 1.49 & 1.12 & 1930 & 10 \\
P74200 & $2+0.0$ & 1.04 & 1.11 & 3060 & 11 \\
P74600 & $2+0.5$ & 1.17 & 1.02 & 2200 & 12 \\
P75800 & $3+0.0$ & 1.13 & 1.06 & 3060 & 13 \\
P76200 & $3+0.5$ & 1.13 & 0.96 & 2270 & 14 \\
P77000 & $4+0.0$ & 1.17 & 1.14 & 2870 & 15 \\
M96400 & $0+0.5$ & 0.93 & 0.92 & 2310 & 16 \\
M97600 & $1+0.0$ & 1.19 & 1.15 & 2750 & 17 \\
M97800 & $1+0.5$ & 0.88 & 0.90 & 2460 & 18 \\
M98800 & $2+0.0$ & 1.23 & 1.19 & 2650 & 19 \\
O64210 & $0+0.5$ & 1.12 & 1.09 & 2050 & 20 \\
O64530 & $0+0.8$ & 0.93 & 0.95 & 2150 & 21 \\
O64700 & $1+0.0$ & 1.05 & 1.01 & 2310 & 22 \\
\hline
\end{tabular}

distance from the star's center at which the Rosseland optical depth equals unity $\left(\tau_{\text {Ross }}=1\right)$. This radius has the advantage of agreeing well (see Table 6 and the discussion in HSW98 for deviations sometimes occurring in very cool stars) with measurable near-infrared continuum radii and with the standard boundary radius of pulsation models with $T_{\text {eff }} \propto\left(L / R^{2}\right)^{1 / 4}$.

Stellar filter radius $R_{\mathrm{f}}$. For the K-band filter used for the observations we have calculated the theoretical CLVs corresponding to the above mentioned five Mira star models at different phases and cycles. The stellar radius for filter transmission $\mathrm{f}_{\lambda}$ is the intensity and filter weighted radius $R_{\mathrm{f}}=\int R_{\lambda} I_{\lambda} \mathrm{f}_{\lambda} d \lambda / \int I_{\lambda} \mathrm{f}_{\lambda} d \lambda$, which we call stellar filter radius $R_{\mathrm{f}}$ after the definition of Scholz \& Takeda ${ }^{19}$. In this equation $R_{\lambda}$ denotes the above monochromatic $\tau_{\lambda}=1$ radius, $I_{\lambda}$ the central intensity spectrum and $f_{\lambda}$ the transmission of the filter. 
Observed angular stellar $K$-band radius $R_{\mathrm{K}, \mathrm{m}}^{a}$ and observed angular Rosseland radius $R_{\mathrm{m}}^{a}$. The observed angular stellar K-band radii $R_{\mathrm{K}, \mathrm{m}}^{a}$ of the observed Miras corresponding to the model-phase combinations m (see Table 3), were derived by least-squares fits between the measured visibilities and the visibilities of the corresponding theoretical CLVs. Additionally, the angular Rosseland radii $R_{\mathrm{m}}^{a}$ were derived from the obtained stellar $\mathrm{K}$-band radii $R_{\mathrm{K}, \mathrm{m}}^{a}$ and the theoretical ratios $R_{\mathrm{m}} / R_{\mathrm{K}, \mathrm{m}}$ from Table 3 (Table 3 provides theoretical $R$ and $R_{\mathrm{K}}$ values for each model-phase combination $\mathrm{m}$ ). In the following subsections we apply CLVs predicted from all five models at phases both near our observations and, for comparison, also at other phases.

\subsection{Effective temperature}

Effective temperatures of each observed Mira star were derived from its angular Rosseland radii $R_{\mathrm{m}}^{a}$ and its bolometric flux using the relation

$$
T_{\text {eff }}=2341 \mathrm{~K} \times\left(F_{\mathrm{bol}} / \phi^{2}\right)^{1 / 4}
$$

where $F_{\mathrm{bol}}$ is the apparent bolometric flux in units of $10^{-8} \mathrm{erg} \mathrm{cm}^{-2} \mathrm{~s}^{-1}$ and $\phi=2 \times R_{\mathrm{m}}^{a}$ is the angular Rosseland diameter in mas. The bolometric flux was derived from JHKLM-band observations carried out twelve days after the visibility observations. For cool stars such as LPVs, where most of the luminosity is emitted at near-infrared wavelengths, a convenient approximation for calculating bolometric magnitudes is to use a blackbody function to interpolate between photometric observations in the $\mathrm{J}, \mathrm{H}, \mathrm{K}, \mathrm{L}$ and $\mathrm{M}$ bands. For estimating the bolometric flux we used JHKLM photometric measurements which were carried out with the $1.25 \mathrm{~m}$ telescope at the Crimean station of the Sternberg Astronomical Institute in Moscow twelve days after our visibility observations.

Fig. 2 shows a comparison of the measured and theoretical effective temperatures. Table 4 lists the measured bolometric flux and the average measured effective temperature for each of the five observed Mira stars. For R Aql we also derived effective temperatures for the best-fitting $\mathrm{D}$ and $\mathrm{P}$ models:
Measured effective temperature of R Aql:
$3007 \pm 155 \mathrm{~K}$
(D model $\left.{ }^{*}\right)$;
$3109 \pm 168 \mathrm{~K}$
$\left(\mathrm{P}\right.$ model $\left.^{* *}\right)$
Theoretical D and P model effective temperature: $3025 \mathrm{~K}$
$3030 \mathrm{~K}$
$\left(\mathrm{P} \operatorname{model}^{* *}\right)$

$\left({ }^{*}\right.$ average over phases $1.0,2.0 ;{ }^{* *}$ average over phases $\left.1.0,2.0,3.0,4.0\right)$

Table 4. Observational data and measured effective temperatures.

\begin{tabular}{|c|c|c|c|c|l|}
\hline Star & Date & $\Phi_{\text {vis }}$ & $\begin{array}{c}K \\
{[\mathrm{mag}]}\end{array}$ & $\begin{array}{c}\mathrm{F}_{\text {bol }} \\
{\left[10^{-8} \mathrm{erg} / \mathrm{cm}^{2} \mathrm{~s}\right]}\end{array}$ & $\begin{array}{l}T_{\text {eff }} \\
{[\mathrm{K}]}\end{array}$ \\
\hline X Oph & 99 May 27 & 0.71 & -0.83 & $320.6 \pm 50.1$ & $2926 \pm 152^{* *}$ \\
R Aql & 99 May 28 & 0.17 & -0.86 & $351.2 \pm 52.7$ & $3072 \pm 161^{*}$ \\
RU Her & 99 May 21 & 0.07 & -0.11 & $159.8 \pm 24.0$ & $2959 \pm 152^{*}$ \\
R Ser & 99 May 21 & 0.28 & 0.02 & $170.2 \pm 25.5$ & $3112 \pm 160^{* *}$ \\
V CrB & 99 May 27 & 0.07 & 0.96 & $52.8 \pm 8.0$ & $2325 \pm 122^{*}$ \\
\hline
\end{tabular}

( ${ }^{*}$ average over all near-maximum model-phase combinations $\mathrm{m}$ since the phase of the observation was near-maximum) $\left({ }^{* *}\right.$ average over all model-phase combinations $\mathrm{m}$ since models with phases close to the observation do not exist)

\subsection{Linear radii}

We have derived linear stellar K-band radii $R_{\mathrm{K}, \mathrm{m}}$ and Rosseland radii $R_{\mathrm{m}}$ of $\mathrm{R}$ Aql from the measured angular stellar K-band radii $R_{\mathrm{K}, \mathrm{m}}^{a}$ and Rosseland radii $R_{\mathrm{m}}^{a}$ by using the $\mathrm{R}$ Aql HIPPARCOS parallax of $4.73 \pm 1.19 \mathrm{mas}^{20}$. The HIPPARCOS parallaxes of the other four observed Miras have too large errors for estimating useful linear radii. Fig. 3 shows the obtained linear Rosseland radii $R_{\mathrm{m}}$ and stellar K-band radii $R_{\mathrm{K}, \mathrm{m}}$ of $\mathrm{R}$ Aql for all model-phase combinations $\mathrm{m}$. The theoretical Rosseland radii of the $\mathrm{D}, \mathrm{M}$ and $\mathrm{P}$ model series at all available near-maximum phases are close (within the error bars) to the measured Rosseland radii of $\mathrm{R} \mathrm{Aql.} \mathrm{The} \mathrm{theoretical} \mathrm{Rosseland} \mathrm{radii}$ of the first-overtone models $\mathrm{E}$ and $\mathrm{O}$ are clearly too large compared with measured Rosseland radii. The same conclusions are also valid for the linear stellar filter radii $R_{\mathrm{K}}$ (Fig. 3).

If we calculate average $\mathrm{R}$ Aql radii by averaging the radii derived with all available near-maximum $\mathrm{D}$ model CLVs (i.e., $\mathrm{m}=1,3$ ) and/or near-maximum $\mathrm{P}$ model CLVs (i.e., $\mathrm{m}=9,11,13,15)$ we obtain: 

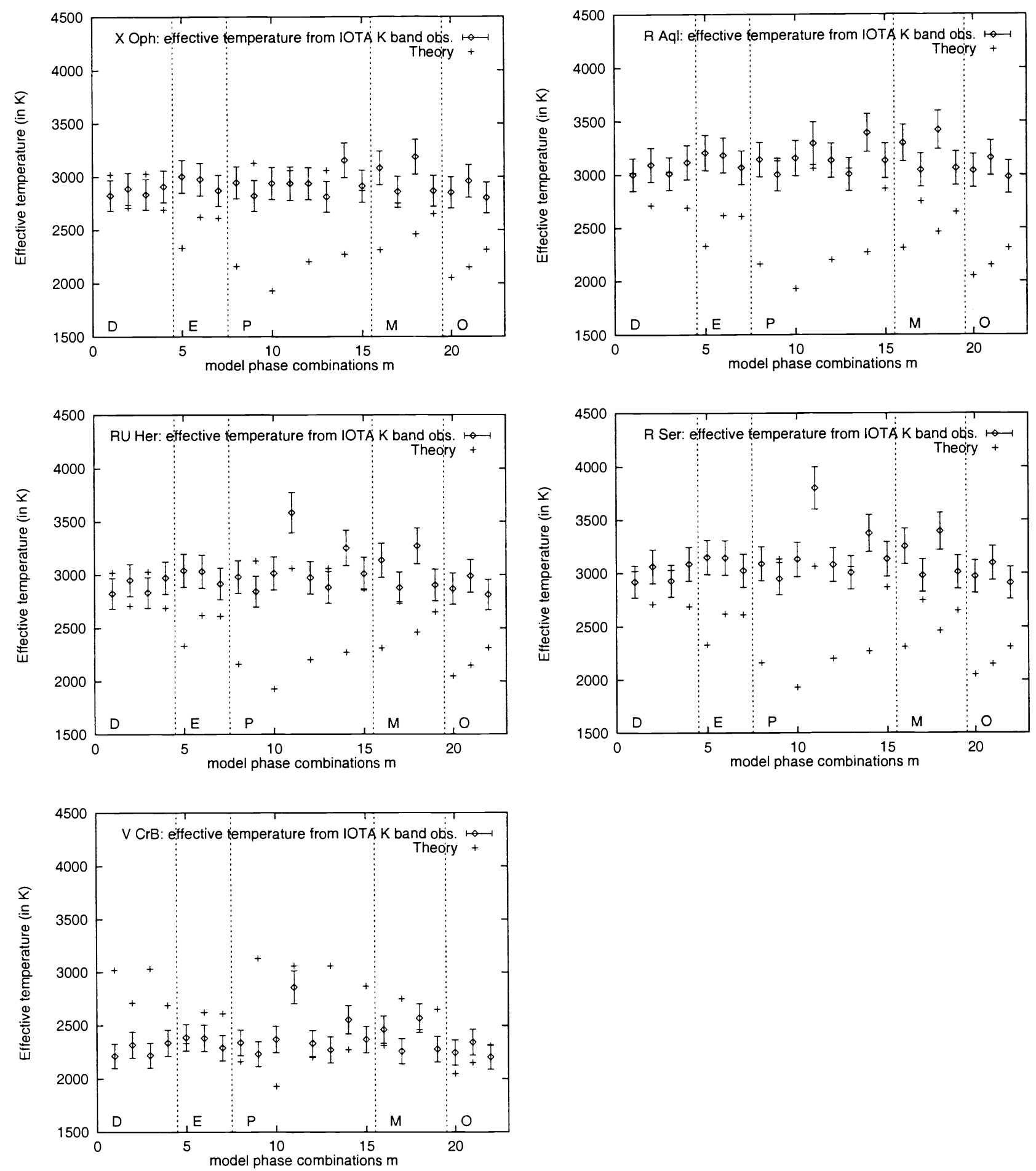

Figure 2. Comparison of measured effective temperatures of the 5 observed Mira stars and the theoretical model effective temperatures (see text). Table 3 shows the link between the abscissa values and the models and their phases. 

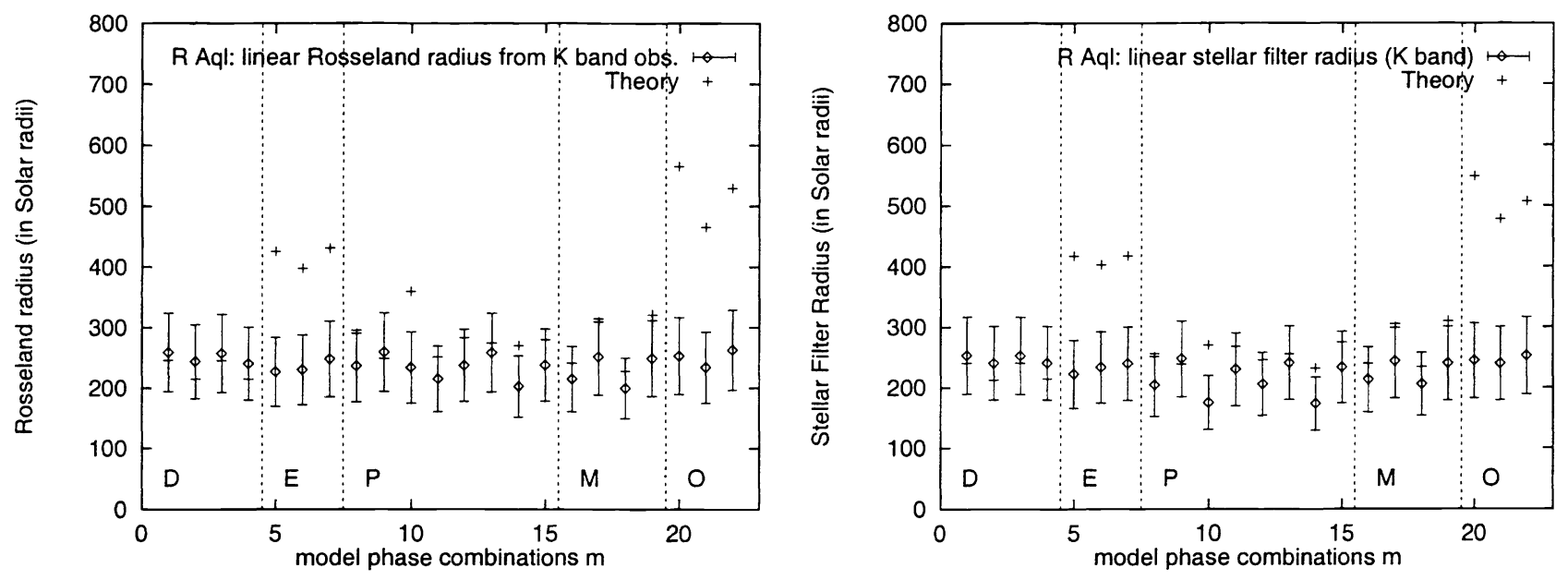

Figure 3. Comparison of measured $\mathrm{R}$ Aql radii and theoretical model radii: (left) linear Rosseland radii $R_{\mathrm{m}}$ and (right) linear stellar K-band radii $R_{\mathrm{K}, \mathrm{m}}$ for all 22 model-phase combinations $\mathrm{m}$. Table 3 gives the link between the abscissa values (model-phase combinations $\mathrm{m}$ ) and the models and their phases.

Average theoretical D model Rosseland radius:

Average measured $\mathrm{D}$ model $\mathrm{R}$ Aql Rosseland radius:

Average theoretical $\mathrm{P}$ model Rosseland radius:

Average measured $\mathrm{P}$ model $\mathrm{R}$ Aql Rosseland radius:

Average theoretical $\mathrm{D} / \mathrm{P}$ model Rosseland radius:

Average measured $\mathrm{D} / \mathrm{P}$ model $\mathrm{R}$ Aql Rosseland radius:
$246 \mathrm{R}_{\odot}$

$258 \mathrm{R}_{\odot} \pm 65 \mathrm{R}_{\odot} \quad$ (obtained with $\mathrm{m}=1$ and 3 )

$263 \mathrm{R}_{\odot}$

$242 \mathrm{R}_{\odot} \pm 61 \mathrm{R}_{\odot} \quad$ (obtained with $\mathrm{m}=9,11,13$ and 15$)$

$255 \mathrm{R}_{\odot}$

$250 \mathrm{R}_{\odot} \pm 63 \mathrm{R}_{\odot} \quad$ (obtained with $\mathrm{m}=1,3,9,11,13,15$, 17, 19; D and P models)

\subsection{Pulsation mode}

Adopting the above phase-averaged (over models $\mathrm{D}$ and $\mathrm{P}$ at maximum phases) linear Rosseland radius of $250 \mathrm{R}_{\odot} \pm 63 \mathrm{R}_{\odot}$ for $\mathrm{R} \mathrm{Aql}$, we find for the pulsation constant $Q=P\left(M / M_{\odot}\right)^{1 / 2}\left(R / R_{\odot}\right)^{-3 / 2}$ a value of $Q=0.072 \pm 0.027$ for a $1 \mathrm{M}_{\odot-}$ Mira with period $P=284$ days. This $Q$ value agrees within the $1 \sigma$ error with the theoretical value $(Q=0.088)$ for fundamental pulsation mode for $1 \mathrm{M}_{\odot}$-AGB stars with a period of $\sim 284$ days ${ }^{21}$. The corresponding $Q$ value of first overtone pulsation mode is $Q=0.049$. Note, however, that no direct measurement of a Mira mass exists and that a $20 \%$ uncertainty of $M$ would for example result in a $10 \%$ uncertainty of $Q$.

\section{DISCUSSION}

We derived the following angular uniform-disk diameters $\phi$ of five Mira stars from K-band visibility measurements with the $38 \mathrm{~m}$ baseline of the IOTA interferometer and the FLUOR beam combiner:

$$
\begin{array}{ll}
\text { X Oph: } & \phi=11.7 \mathrm{mas} \pm 0.3 \mathrm{mas} \\
\text { R Aql: } & \phi=10.9 \mathrm{mas} \pm 0.3 \mathrm{mas} \\
\text { RU Her: } & \phi=8.4 \mathrm{mas} \pm 0.2 \mathrm{mas} \\
\text { R Ser: } & \phi=8.1 \mathrm{mas} \pm 0.2 \mathrm{mas} \\
\text { V CrB: } & \phi=7.9 \mathrm{mas} \pm 0.2 \mathrm{mas}
\end{array}
$$

The following effective temperatures were obtained from photometric JHKLM observations and the derived angular Rosseland radii: 


$$
\begin{array}{lll}
\text { X Oph } & \left(\Phi_{\text {vis }}=0.71\right): & 2926 \mathrm{~K} \pm 152 \mathrm{~K} \\
\text { R Aql } & \left(\Phi_{\text {vis }}=0.17\right): & 3072 \mathrm{~K} \pm 161 \mathrm{~K} \\
\text { RU Her } & \left(\Phi_{\text {vis }}=0.07\right): & 2959 \mathrm{~K} \pm 152 \mathrm{~K} \\
\text { R Ser } & \left(\Phi_{\text {vis }}=0.28\right): & 3112 \mathrm{~K} \pm 160 \mathrm{~K} \\
\text { V CrB } & \left(\Phi_{\text {vis }}=0.07\right): & 2325 \mathrm{~K} \pm 122 \mathrm{~K}
\end{array}
$$

Previous interferometric K-band observations of some of our target stars (R Aql, X Oph, R Ser) were carried out by van Belle et al. ${ }^{8}$ at similar phases. Their derived uniform-disk diameters $\left(\mathrm{R} \mathrm{Aql} ; \Phi_{\mathrm{vis}}=0.90: 10.76 \pm 0.61 \mathrm{mas}\right.$, $\mathrm{X} \mathrm{Oph} ; \Phi_{\mathrm{vis}}=0.75: 12.30 \pm 0.66 \mathrm{mas}, \mathrm{R} \mathrm{Ser} ; \Phi_{\mathrm{vis}}=0.32: 8.56 \pm 0.58 \mathrm{mas}$ ) are in good agreement with our observations (within the error bars). Their measured effective temperatures (R Aql: $3189 \pm 147 \mathrm{~K}, \mathrm{X}$ Oph: $3041 \pm 160 \mathrm{~K}$, R Ser: $2804 \pm 144 \mathrm{~K}$ ) are also in good agreement with our results, with the exception of $\mathrm{R}$ Ser.

For R Aql a good HIPPARCOS parallax (4.73 \pm 1.19 mas) is available and it is therefore possible to compare measured linear Rosseland and stellar K-band radii with the theoretical radii of the BSW96 and HSW98 models. The measured radii were derived by fitting theoretical (BSW96, HSW98) center-to-limb intensity variations to the visibility data. In the following table we compare measured and theoretical values:

$\begin{array}{lllll}\text { Measured linear R Aql Rosseland radii } R_{\mathrm{m}}: & 258 \pm 65 \mathrm{R}_{\odot} & \left(\mathrm{D} \text { model }^{*}\right) ; & 242 \pm 61 \mathrm{R}_{\odot} & \left(\mathrm{P} \text { model }^{* *}\right) \\ \text { Theoretical linear Rosseland radii } R_{\mathrm{m}}: & 246 \mathrm{R}_{\odot} & \left(\mathrm{D} \text { model }^{*}\right) ; & 263 \mathrm{R}_{\odot} & \left(\mathrm{P} \text { model }^{* *}\right) \\ \text { Measured linear R Aql stellar K-band radii } R_{\mathrm{K}, \mathrm{m}}: & 253 \pm 64 \mathrm{R}_{\odot} & \left(\mathrm{D} \text { model }^{*}\right) ; & 238 \pm 61 \mathrm{R}_{\odot} & \left(\mathrm{P} \text { model }^{* *}\right) \\ \text { Theoretical linear stellar K-band radii } R_{\mathrm{K}, \mathrm{m}}: & 241 \mathrm{R}_{\odot} & \left(\mathrm{D} \text { model }^{*}\right) ; & 259 \mathrm{R}_{\odot} & \left(\mathrm{P} \text { model }^{* *}\right) \\ \text { Measured R Aql effective temperature: } & 3007 \pm 155 \mathrm{~K} & \left(\mathrm{D} \text { model }^{*}\right) ; & 3109 \pm 168 \mathrm{~K} & \left(\mathrm{P} \text { model }^{* *}\right) \\ \text { Theoretical effective temperature: } & 3025 \mathrm{~K} & \left(\mathrm{D} \text { model }^{*}\right) ; & 3030 \mathrm{~K} & \left(\mathrm{P} \text { model }^{* *}\right)\end{array}$

( ${ }^{*}$ average over phases $1.0,2.0 ;{ }^{* *}$ average over phases $\left.1.0,2.0,3.0,4.0\right)$

The comparison suggests that R Aql is well represented by the fundamental mode D and P model (BSW96, HSW98). The measured Rosseland radius of $R=250 \pm 63 \mathrm{R}_{\odot}$ (average of the derived values from $\mathrm{D}$ and $\mathrm{P}$ model CLVs; corresponding theoretical $\mathrm{D} / \mathrm{P}$ model Rosseland radius $=255 \mathrm{R}_{\odot}$ ) places $\mathrm{R}$ Aql among the fundamental mode pulsators in the period-radius relation which also is in agreement with Ref. 8. Note, however, that observations in more filters than just one continuum filter may be necessary for safely distinguishing a well-fitting model from an accidental match (cf. Ref. 10).

\section{REFERENCES}

1. M.S. Bessell, M. Scholz, P.R. Wood, $A \& A$ 307, pp. 481, 1996 (BSW96)

2. K.-H. Hofmann, M. Scholz, P.R. Wood, $A \& A$ 339, pp. 846, 1998 (HSW98)

3. D. Bonneau, A. Labeyrie, $A p J$ 181, pp. L1, 1973

4. M. Karovska, P. Nisenson, C. Papaliolios, R.P. Boyle, ApJ 374, pp. L51, 1991

5. A. Quirrenbach, D. Mozurkewich, J.T. Armstrong, et al., $A \& A$ 259, pp. L19, 1992

6. C.A. Haniff, M. Scholz, P.G. Tuthill, MNRAS 276, pp. 640,1995

7. G. Weigelt, Y. Balega, K.-H. Hofmann, M. Scholz, A\&A 316, pp. L21, 1996

8. G.T. Van Belle, H.M. Dyck, J.A. Benson, M.G. Lacasse, $A J$ 112, pp. 2147, 1996

9. G. Perrin, V. Coudé du Foresto, S.T. Ridgway, et al., $A \& A A 345$, pp. 221, 1999

10. K.-H. Hofmann, Y. Balega, M. Scholz, G. Weigelt, $A \& A$ 353, pp. 1016, 2000

11. T. Watanabe, K. Kodaira, PASJ 31, pp. 61, 1979

12. M. Scholz, $A \varangle A$ 145, pp. 251, 1985

13. M.S. Bessell, J.M. Brett, M. Scholz, P.R. Wood, $A \& A$ 213, pp. 209, 1989

14. N.P. Carleton, W.A. Traub, M.G. Lacasse, et al., Proc. SPIE 2200, pp. 152, 1994

15. W.A. Traub, et al., Proc. SPIE 3350, pp. 848, 1998

16. V. Coudé du Foresto, S.T. Ridgway, J.-M. Mariotti, $A \mho A S$ 121, pp. 379, 1997

17. G. Perrin, V. Coudé du Foresto, S.T. Ridgway, et al. $A \& B$ 331, pp. 619, 1998

18. H.M. Dyck, J.A. Benson, G.T. Van Belle, S.T. Ridgway, $A J$ 111(4), pp. 1705, 1996

19. M. Scholz, Y. Takeda, $A \& A$ 186, pp. 200, 1987 (erratum: 196, 342)

20. F. Van Leeuwen, M.W. Feast, P.A. Whitelock, B. Yudin, MNRAS 287, pp. 955, 1997

21. M.W. Fox, P.R. Wood, ApJ 259, pp. 198, 1982 